\title{
Oncological and renal outcomes of segmental ureterectomy vs. radical nephroureterectomy for upper tract urothelial carcinoma
}

\author{
TOMONORI KATO ${ }^{1,2^{*}}$, RYO NAKAYAMA ${ }^{1 *}$, TOMOMI HABA ${ }^{1}$, MAKOTO KAWAGUCHI ${ }^{3}$, \\ AKIRA KOMIYA ${ }^{4}$ and HIROSHI KOIKE ${ }^{1}$

\begin{abstract}
${ }^{1}$ Division of Urology, Niigata Rosai Hospital, Japan Organization of Occupational Health and Safety, Joetsu, Niigata 942-8502; ${ }^{2}$ Department of Urology, Teikyo University Chiba Medical Center, Ichihara, Chiba 299-0111; ${ }^{3}$ Division of Diagnostic Pathology, Niigata Rosai Hospital, Japan Organization of Occupational Health and Safety, Joetsu, Niigata 942-8502; ${ }^{4}$ Department of Urology, Chiba University Graduate School of Medicine, Chiba, Chiba 260-8670, Japan
\end{abstract}

Received December 28, 2017; Accepted August 31, 2018

DOI: $10.3892 / \mathrm{ol} .2018 .9463$

\begin{abstract}
Segmental ureterectomy (SU) represents a promising alternative for the treatment of upper tract urothelial carcinomas (UTUCs) as it is a less invasive procedure and guarantees the preservation of renal units. The present study evaluated oncological outcomes and renal functions following SU when compared with radical nephroureterectomy (RNU). A total of 26 patients with UTUCs who underwent SU $(n=12)$ or RNU ( $n=14)$ were retrospectively evaluated. SU was performed in patients with clinically unifocal disease. In the SU group, the following surgeries were carried out: 7 direct ureterocystoneostomy, 1 reimplantation on psoas hitch bladder, 1 reimplantation on Boari flap bladder, 2 ureteral end-to-end anastomosis and 1 subtotal ureterectomy. In the SU group, tumors were low grade urothelial carcinoma (UC) in 6 patients, high grade UC in 5 patients and high grade UC with squamous cell differentiation in 1 patient, as well as $\leq \mathrm{pT} 1$ in $5, \geq \mathrm{pT} 2$ in 6 and pTis in 1 patient; ' $\mathrm{p}$ ' refers to the pathological state. The 5-year overall, cancer-specific, recurrence free and metastasis free survival in the SU group were 77.8, 87.5, 34.4 and $80.8 \%$, respectively, which all exhibited no significant differences when compared with those of the RNU group.
\end{abstract}

Correspondence to: Dr Tomonori Kato, Department of Urology, Teikyo University Chiba Medical Center, 3426-3, Anesaki, Ichihara, Chiba 299-0111, Japan

E-mail: jpn3899kato@yahoo.co.jp

${ }^{*}$ Contributed equally

Abbreviations: SU, segmental ureterectomy; RNU, radical nephroureterectomy; UTUC, upper tract urothelial carcinoma; UC, urothelial carcinoma; TURBT, transurethral resection of bladder tumor; CT, computed tomography; MRI, magnetic resonance imaging; eGFR, estimated glomerular filtration rate; SD, standard deviation

Key words: kidney sparing surgery, oncological outcome, RNU, renal function, SU, UTUC
With regard to renal function, postoperative estimated glomerular filtration rates were preserved in the SU group. The present study demonstrated that SU does not result in poorer cancer control when compared with RNU. Thus, SU is an acceptable alternative to RNU in selected cases, as it is less invasive and preserves renal functions.

\section{Introduction}

The aging of Japan's population is advancing, accordingly geriatric patients are often diagnosed to have malignancy. Although radical surgery is appropriate in terms of curativity, conservative procedures are often prefered in such geriatric patients. Since the population in our medical area is also continuing to age, urothelial carcinomas (UCs), which are generally diseases of middle-aged and elderly people, are mainly diagnosed in geriatric patients older than 60 years in our institution.

Radical nephroureterectomy (RNU) is considered to be the standard treatment for patients with non-metastatic upper tract urothelial carcinomas (UTUCs), considering that UTUCs are frequently multifocal and have high ipsilateral recurrence rate $(1,2)$. Meanwhile, segmental ureterectomy (SU) is an interesting alternative for the treatment of UTUCs because it allows a less invasive procedure and guarantees the preservation of renal units.

In the field of renal oncology, it has been emphasized that the post-operative renal insufficiency may lead to higher rates of dialysis, cardiovascular morbidity and overall mortality $(3,4)$. Therefore, partial nephrectomy is currently recommended with respect to small renal masses (5).

Unlike renal tumors, SU for UTUCs has never been popularized yet. One of the reasons is that, UTUCs are relatively uncommon, accounting for only $5-6 \%$ of all UCs $(6,7)$. Accordingly, only limited data are available on the oncological outcomes afforded by conservative management. However, SU has gradually become more acceptable with recent data supporting the importance of nephron-sparing. In our institution, SU for UTUCs has been performed over the last decade. In this study, we retrospectively analyzed the data of patients treated with $\mathrm{SU}$ in comparison with patients treated with 
RNU. Through this study, we would like to introduce this kidney-sparing procedures to nephrologists and urologists.

\section{Patients and methods}

Patients. The subjects are comprised with consecutive 26 Japanese patients who had localized ipsilateral UTUCs who were treated with SU or RNU between January 2004 and December 2016. These 26 patients were retrospectively divided into 2 groups according to therapy: The SU group $(\eta=12)$, patients treated with SU; and the RNU group $(\eta=14)$, patients treated with RNU.

These patients had no previous history of radical cystectomy for bladder tumor. Four patient in the SU group and 1 patient in the RNU group had previous histories of superficial bladder tumor which had been curatively treated with transurethral resection of bladder tumor (TURBT). No patients had co-existence of bladder tumors at the time of SU or RNU. All these patients had no metastatic disease at the time of diagnosis.

The present study conformed to the principles outlined in the Declaration of Helsinki. The Ethics Committee of Niigata Rousai Hospital (Niigata, Japan) approved the study (cat. no. 2017-01) and waived the requirement for approval and informed consent for patient participation in this study due to the retrospective nature of this analysis of clinical data. The present study was conducted using the opt-out method.

Treatment. Both SU and RNU were all performed through open surgery. SU was performed to patients with clinically unifocal disease without evidence of invasion in the preoperative imaging. Surgical methods for the SU group patients depend on the tumor location. For distal tumors, distal ureterectomy with a bladder cuff resection plus ureteral re-implantation was carried out. As re-implantation, direct ureterocystoneostomy was mostly performed. When ureteral length is insufficient for direct re-implantation, a Boari bladder flap or a psoas bladder hitch were then used depending on the surgeon's preference. Meanwhile, for the mid or proximal tumors, partial ureterectomy with ureteral end-to-end anastomosis was performed. On the other hand, RNU with a bladder cuff resection was performed in the patients for which SU may not be appropriate, such as invasive and/or multifocal UTUCs and/or renal pelvic tumors. Of 14 patients in the RNU group, 2 patients had radiologically invasive disease. All patients were diagnosed as clinically N0 disease for both SU and RNU, therefore abdominal or pelvic lymph node dissection was not performed during this period.

Preoperatively, preoperative $\mathrm{T}$ stratification was established by Computed tomography (CT) and/or Magnetic Resonance Imaging (MRI). Upper tract urinary cytology was performed as part of a standard diagnostic work-up, and all patients were diagnosed to have UCs preoperatively. Diagnostic ureteroscopy with biopsy was not routinely practiced during this period. All histological examinations were performed at the Institute of the Pathology at our hospital. Tumors were staged according to the 2002 International Union Against Cancer TNM classification, clinical stage and pathological stage are denoted by a small ' $c$ ' or ' $p$ ' before the stage (8). Tumor grading was assessed according to the
1998 WHO/International Society of Urological Pathology consensus classification (9).

Follow-up procedures. Follow-up consisted of routine blood work and serum chemistry studies, urinary cytology, cystoscopy, ultrasound sonography and CT scan. Cystoscopy and urinary cytology were examined every 3 months up to the 5th year, then every 6 months thereafter. Ultrasound sonography was done every 6 months up to the 5th year, and annually thereafter. A CT evaluating the chest and abdomen was performed annually, and additionally done when clinically indicated. Elective bone scan and MRI were also performed when clinically indicated.

When the recurrence without distant metastasis has developed, curative surgery was added whenever possible. TURBT was performed when bladder only recurrences occurred. Radical cystectomy was subsequently carried out when the bladder recurrence proved to be invasive disease. If ipsilateral localized recurrence occurred, RNU for the residual kidney and ureter was performed.

Statistical analysis. Statistical analysis of the data was performed using StatView version 5 software (SAS Institute, Inc., Cary, NC, USA). The significance of any differences in patient characteristics among groups was tested using Student's t-test or Mann-Whitney U test. Statistical comparison of estimated glomerular filtration rates (eGFRs) between groups was made by two-way repeated measure analysis of variance (ANOVA) with Fisher's protected least significant difference (PLSD) post hoc test. Repeated measure one-way ANOVA followed fisher's PLSD for multiple comparisons, in which each value was compared with the preoperative control value, was also used.

The survival curves were determined using the Kaplan-Meier method, and differences were evaluated using the log-rank test. A univariate Cox regression model was used to evaluate the risk factors. $\mathrm{P}<0.05$ was considered to indicate a statistically significant difference.

\section{Results}

Patient characteristics. Clinical and pathological characteristics are shown in Table I. Mean age at diagnosis was 72.5 (range, 62-78) years for the SU group and 73.7 (range, 65-82) years for the RNU group, respectively. No significant differences between treatment groups were observed with respect to age, sex, affected side, previous bladder cancer history and tumor multiplicity. Meanwhile, patients treated with SU tend to have smaller tumor when compared to patients treated with RNU $(20.6 \pm 9.7 \mathrm{~cm}$ vs. $33.4 \pm 19.7 \mathrm{~cm}, \mathrm{P}=0.05)$. And there was obvious difference in tumor locations between the two groups $(\mathrm{P}<0.01)$. It is because RNUs are indicated for all site of tumors including renal pelvic tumors, for which $\mathrm{SU}$ is scarcely indicated.

Among the SU group, 11 of 12 patients had elective indications with normal contralateral kidney and the remaining 1 patient had imperative indication. The only imperative case was that of a 74 year old male who had a solitary left kidney due to past right nephrectomy for renal tuberculosis. He was presented with acute renal failure due to obstructive 
Table I. Subject characteristics.

\begin{tabular}{|c|c|c|c|}
\hline Characteristic & SU group & RNU group & P-value \\
\hline Total $\mathrm{n}$ & 12 & 14 & \\
\hline Age, years [mean $\pm \mathrm{SD}$ (range)] & $72.5 \pm 4.4(62-78)$ & $73.7 \pm 5.8(65-82)$ & 0.54 \\
\hline Sex & & & 0.76 \\
\hline Male & 7 & 9 & \\
\hline Female & 5 & 5 & \\
\hline Affected side & & & 0.99 \\
\hline Righ & 6 & 7 & \\
\hline Left & 6 & 7 & \\
\hline Tumor location & & & $<0.01$ \\
\hline Renal pelvis & 0 & 8 & \\
\hline Upper ureter & 1 & 1 & \\
\hline Middle ureter & 2 & 3 & \\
\hline Distal ureter & 9 & 1 & \\
\hline Throughout upper tract & 0 & 1 & \\
\hline Tumor length, mm [mean \pm SD (range)] & $20.6 \pm 9.7(6-35)$ & $33.4 \pm 19.7(10-84)$ & 0.05 \\
\hline Tumor multiplicity & & & 0.18 \\
\hline Solitary & 12 & 12 & \\
\hline Multiple & 0 & 2 & \\
\hline Previous bladder cancer history & & & 0.09 \\
\hline No & 8 & 13 & \\
\hline Yes & 4 & 1 & \\
\hline Clinical tumor stage & & & 0.18 \\
\hline$\leq \mathrm{cT} 2$ & 12 & 12 & \\
\hline$\geq \mathrm{cT} 3$ & 0 & 2 & \\
\hline Pathological tumor stage & & & 0.91 \\
\hline pTa & 4 & 4 & \\
\hline pT1 & 1 & 4 & \\
\hline pT2 & 3 & 1 & \\
\hline pT3 & 3 & 4 & \\
\hline pTis & 1 & 1 & \\
\hline Tumor grade & & & 0.83 \\
\hline Low grade & 6 & 7 & \\
\hline High grade & 5 & 7 & \\
\hline High grade with squamous differentiation & 1 & 0 & \\
\hline
\end{tabular}

c, clinical; p, pathological; SU, segmental ureterectomy; RNU, radical nephroureterectomy; SD, standard deviation.

hydronephrosis in the solitary kidney. Emergently, percutaneous nephrostomy was inserted in order to preserve renal function. He was then diagnosed with proximal ureter cancer. Subsequently, subtotal ureterectomy with ureteropelvic junction ligation was carried out, and permanent nephrostomy was required thereafter.

Among the patients in the SU group, the tumor location was in the distal ureter in 9 of 12 patients $(75 \%)$, followed by the middle ureter in 2 patients (16.6\%) and proximal ureter in 1 patient (8.3\%). In 9 patients with the distal tumor, distal ureterectomy was performed and the following re-implantation was carried out, and divided as follows: direct ureterocystoneostomy, 7 patients; reimplantation on psoas hitch bladder, 1 patient; reimplantation on Boari flap bladder, 1 patient. Meanwhile, SU with ureteral end-to-end anastomosis was performed in 2 patients with mid ureter tumors. The remaining one patient with proximal tumor underwent permanent nephrostomy plus subtotal ureterectomy as stated above.

Pathological findings. In the SU group, tumors were low grade UC in $6(50.0 \%)$, high grade UC in 5 (41.7\%), and high grade UC with squamous cell differentiation in $1(8.3 \%)$ patient, as well as $\leq \mathrm{pT} 1$ in $5(41.7 \%), \geq \mathrm{pT} 2$ in $6(50.0 \%)$ and pTis in 1 patient $(8.3 \%)$. While in the RNU group, tumors were low grade UC in 7 $(50.0 \%)$ and high grade UC in $7(50.0 \%)$, as well as $\leq \mathrm{pT} 1$ in 8 $(57.1 \%), \geq$ pT 2 in $5(35.7 \%)$ and pTis in 1 patient $(7.1 \%)$. 
Survival. Univariate Cox regression analysis of prognostic factors for the overall survival (OS), cancer-specific survival (CSS), recurrence-free survival (RFS) and metastasis free survival (MFS) are shown in Table II. Although the sample size is small, patients with high grade and/or invasive tumors had significantly worse OS rates than patients with low grade and stage tumors. There also was a trend towards lower CSS and MFS, which did not reach significance. On the other hand, there is no obvious difference in these survival rates between the $\mathrm{SU}$ group and the RNU group.

Mean follow-up period was 48.5 (range, 7-148) months for the SU group and 46.9 (range, 6.0-122) months for the RNU group, respectively. Fig. 1 demonstrate the OS, CSS, RFS and MFS for patients treated with SU or RNU. The 5-year OS, CSS, RFS and MFS in the SU group were 77.8, 87.5, 34.4 and $80.8 \%$, respectively, which all showed no significant differences compared with those of the RNU group. The 5-year OS, CSS, RFS and MFS in the RNU group were 60.1, $71.9,50.0$ and $73.5 \%$, respectively.

The incidence of recurrence and/or metastasis was similar between the patients undergo SU and RNU. In the SU group, recurrences and/or metastasis were observed in 7/12 cases during the follow-up period. For details, bladder recurrences, upper urinary tract recurrences, lymph node metastasis and visceral metastasis occur in 7,2,2 and 2 cases, respectively. While those were observed in 7/14 cases in the RNU group. For details, bladder recurrences, local recurrences, lymph node metastasis and visceral metastasis occur in 6,1,2 and 3 cases, respectively.

In the SU group, recurrences and/or metastasis did not occur in 5 cases during the follow-up period. Meanwhile, although 4 patients in the SU group developed recurrences, they were all successfully treated with adjuvant surgical procedures and have become free of disease at the time of their last follow-up as stated below. Bladder-only recurrence was found in 3 of these 4 patients. Among them, 2 cases who developed superficial bladder recurrence had undergone curative TURBT, while one who developed invasive bladder recurrence was curatively treated with radical cystectomy. The other one patient experienced repeated superficial bladder recurrence treated with several TURBT and consequently ipsilateral ureteral recurrence appeared 9 years after $\mathrm{SU}$ which curatively treated with RNU for residual urinary tract. In summary, UTUCs were well-controlled in these 9 (75\%) cases in the SU group.

On the other hand, 3 of the 12 patients in the SU group have developed the metastatic disease and, subsequently, 2 of them died from the disease. One patient who died was 70 years old at diagnosis, female and initially treated with right SU plus ureteral end-to-end anastomosis for a mid-ureter tumor, which proved to be pT1 high grade UC. The ipsilateral ureteral recurrence was confirmed at 9 months after SU which was re-operated with RNU for residual urinary tract. After a while, at 61 months after SU, invasive bladder recurrence occurred and she died from the disease at 86 months after initial SU. The other patient who died was 75-years at diagnosis, male and initially treated with right distal ureterectomy for a distal tumor, proved to be a pT3 high grade UC. Left pelvic lymph node metastasis had occurred at 20 months after SU and died from the disease at 35 months after SU. The remaining one patient who developed distant

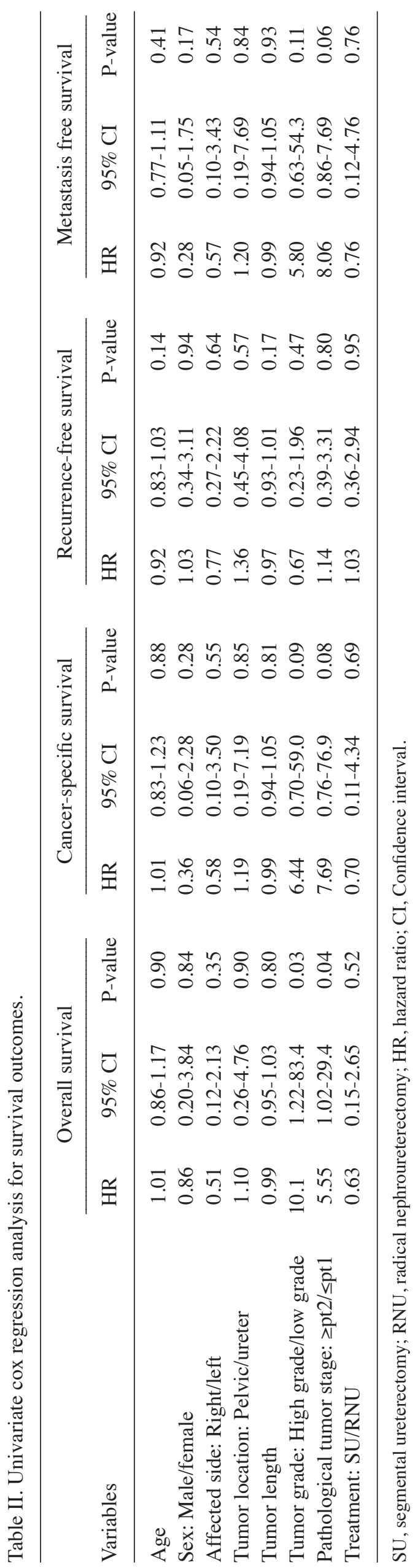


A
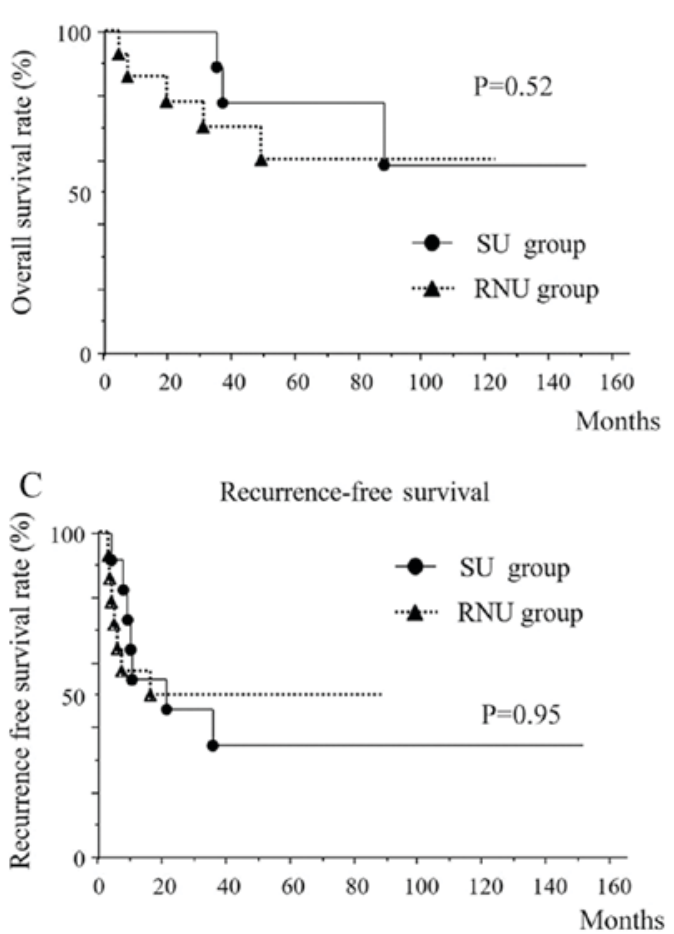

B

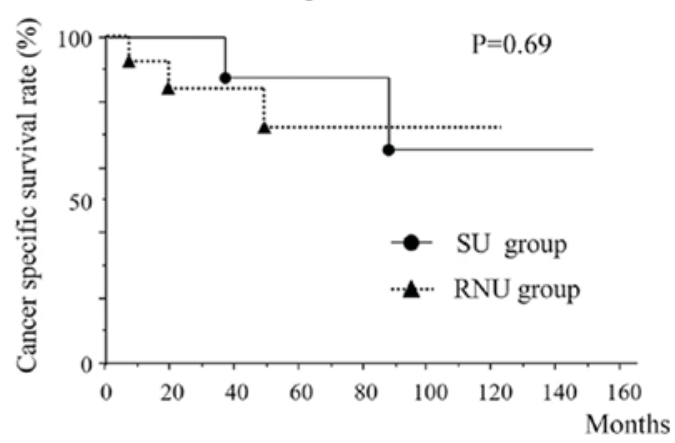

$\mathrm{D}$

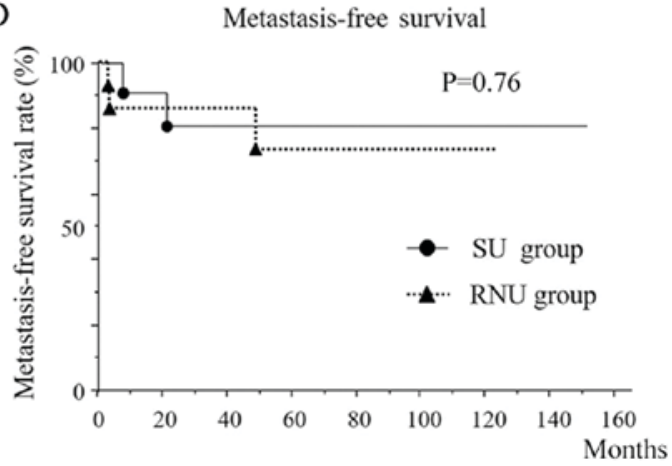

Figure 1. Patient survival. (A) 5-year overall, (B) cancer-specific, (C) recurrence free and (D) metastasis free survival for patients treated with SU or RNU. The 5-year overall, cancer-specific, recurrence free and metastasis free survival in the SU group were 77.8, 87.5, 34.4 and 80.8\%, respectively, which all exhibited no significant differences when compared with those of the RNU group. SU, segmental ureterectomy; RNU, radical nephroureterectomy.

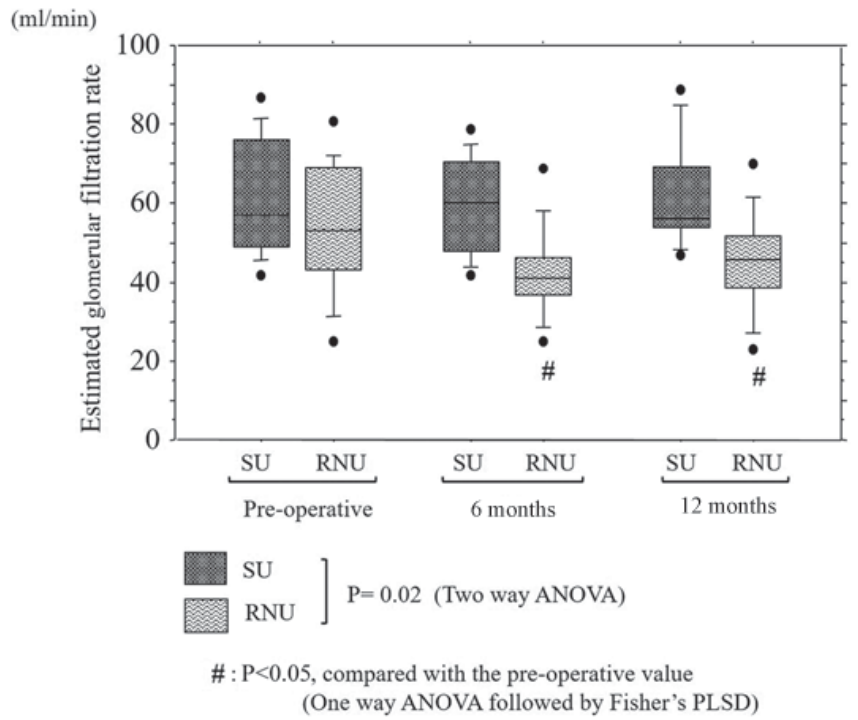

Figure 2. Time course of the changes in the estimated glomerular filtration rates in the SU and RNU groups. Postoperative estimated glomerular filtration rates were well preserved in the $\mathrm{SU}$ group. ${ }^{*} \mathrm{P}<0.05$ vs. pre-operative value. SU, segmental ureterectomy; RNU, radical nephroureterectomy; ANOVA, analysis of variance.

metastasis was 62 years old at diagnosis, female and initially treated with right distal ureterectomy for a distal tumor, proved to be a pT3 high grade UC with positive surgical margin. Multiple liver metastasis had occurred at 7 months after SU. Thereafter, chemotherapy with methotrexate, vinblastine, adriamycin and cisplatin was initiated at the last study follow-up date.
Renal function. Time course of the changes in eGFRs in the $\mathrm{SU}$ and RNU group are shown in Fig. 2. Mean preoperative serum eGFRs were $61.1 \pm 14.2 \mathrm{ml} / \mathrm{min}$ in the $\mathrm{SU}$ group and $52.2 \pm 16.3 \mathrm{ml} / \mathrm{min}$ in the RNU group, with no significant differences between groups $(\mathrm{P}=0.19)$.

In the $\mathrm{SU}$ group, mean postoperative serum eGFRs were $59.8 \pm 12.1$ and $61.6 \pm 13.9 \mathrm{ml} / \mathrm{min}$ at 6 and 12 months, respectively, which showed no significant decrease from the preoperative eGFRs. While in the RNU group, mean postoperative serum eGFRs were $42.3 \pm 11.4$ and $45.1 \pm 12.1 \mathrm{ml} / \mathrm{min}$ at 6 and 12 months, respectively, both of which were lower than the preoperative eGFRs $(\mathrm{P}<0.01$ and $\mathrm{P}=0.02$, respectively).

\section{Discussion}

For non-metastatic UTUCs, RNU is still the standard treatment, which generally results in loss of renal function. It is demonstrated that patients who underwent nephrectomy experienced renal insufficiency with a reduction in mean eGFR by $24 \%(10,11)$. The solitary kidney status is suggested to induce renal insufficiency, higher rates of dialysis, cardiovascular morbidity and overall mortality $(3,4)$. With such a background, conservative therapy has gradually developed. Conservative approaches for UTUCs including SU have originally been developed in patients with imperative indications, such as chronic renal insufficiency, solitary kidneys, or comorbidity $(12,13)$. Gradually, SU has become the most considered alternative procedure for patients who has elective indications with normal contralateral kidney $(1,14,15)$. It is partly because the selection of candidates for SU become safer than before with recent developments of imaging techniques 
which plays important roles in characterizing UTUCs preoperatively $(16,17)$. Needless to say, renal function was well preserved in the SU group. Preservation of renal function may protect patients from non-cancer-related mortality (3). In addition, it may allow the administration of adjuvant chemotherapy for patients who has borderline renal function which may not be administrated once RNU is performed (18).

With regard to oncological outcomes, previous reports retrospectively demonstrated that the incidence of local recurrence was similar between the patient who underwent RNU and distal ureterectomy, although relatively small number of patients were involved (19). The recurrence rate of SU for low stage and low grade UTUCs was estimated at between 10 and $25 \%(20,21)$. Recently, retrospective multicentre study of 2299 patients showed no significant difference in survival between patients treated with SU compared with those treated with RNU (22). Subsequently, Bagrodia et al (23) analyzed a large cohort of 835 patients with UTUC in detail and concluded that partial ureterectomy was not an independent risk factor for recurrence or disease-specific survival on multivariable regression analyses. More recently, Huang et al (24) investigated the oncological and renal outcomes after SU $(n=24)$ and RNU $(n=39)$ who had at least one high-risk factor and demonstrated similar oncological outcomes between SU and RNU, with better preservation of renal function after SU. Our series also demonstrated similar oncologic and renal outcomes to these literatures. SU seems to have comparable oncologic outcomes to RNU, with better preservation of renal function.

In our study, 3 of 12 patients in the SU group had developed metastatic disease and 2 of them died from the disease. These 3 patients had high grade UC and 2 of which had $\geq \mathrm{pT} 3$ disease. It is probable that they could be cured with appropriate surgery (RNU). Preoperative exact diagnosis for appropriate indication for surgery is really needed. Since recent ureteroscopy has good quality and can be performed easily, histological evaluation by ureteroscopic biopsy may be useful.

With regard to tumor location of UTUCs, distal tumors which accounts for almost $70 \%$ are most common than mid and proximal ureter tumors (25). Distal tumors are more frequently solitary, smaller and of lower stage and grade than upper UTUCs $(2,26)$. Distal tumors are also known to be less frequently associated with local recurrence than upper UTUCs (27). Moreover, recurrences tend to be lower stage and grade, as well as occur distal to the primary tumor site in conservatively treated patients $(15,28,29)$. Based on these findings, distal ureterectomy is thought to be the most safe and acceptable option among various conservative therapies $(2,15)$. The open procedure is thought to be the standard for distal ureterectomy so far. However, laparoscopic or robotic distal ureterectomy has been currently developed $(1,30)$.

It is remarkable that our study population comprised geriatric patients aged 62 years and above. For the elderly patients with shorter life expectancies, radical surgery may have minimal impact on eventual outcomes. For geriatric patients, less invasive surgical options should be developed and cancer control should not be considered of supreme importance.

The limitations of this study include its retrospective design, being performed at a single center using a single arm, and the relatively small number of patients. The results may have been biased by the patient selection for SU or RNU. Especially, substantial selection bias for SU group might be present which cannot be overlooked due to our positive recommendation for patients with solitary UTUCs. The results could also have been biased by a long period of time to accrue 2004 to 2016. During the last decade, imaging techniques and diagnostic endoscopic managements have expanded considerably which play important roles in selecting patients. Our series include previous cases wherein imaging techniques were not well improved yet. Moreover, reimplantation on psoas hitch bladder or on Boari flap bladder were used in 2 patients in our series, which may lead difficulty in detecting recurrence via cystoscopy or cause impossibility in performing TURBT. It may result to secondary effect on oncologic outcome. Nevertheless, even with these limitations, the current results suggest that SU is an acceptable alternative for low grade, non-invasive ureteric tumors in selected patients. Further prospective studies with large populations are necessary in order to clarify this issue, which is difficult to conduct due to the low incidence rate of ureteric tumors.

This study demonstrates the efficacy of SU for UTUCs in geriatric patients. SU can preserve renal function and is thought to be an acceptable alternative to RNU in selected patients. We believe that the results of this study could provide useful information on geriatric oncology.

\section{Acknowledgements}

Not applicable.

\section{Funding}

No funding was received.

\section{Availability of data and materials}

The datasets used and/or analyzed during the current study are available from the corresponding author on reasonable request.

\section{Authors' contributions}

TK and RN designed the study, contributed to the analysis and interpretation of data, and wrote the initial draft of the manuscript. TH, MK, AK and HK contributed to the analysis and assisted in the preparation of the manuscript. MK performed the pathological diagnoses. All authors contributed to clinical management of the reported case. All authors critically reviewed the manuscript and approved the final version of the manuscript.

\section{Ethics approval and consent to participate}

The Ethics Committee of Niigata Rousai Hospital approved the present study (cat. no. 2017-01) and waived the requirement for approval and written informed consent for patient participation in the study due to the retrospective nature of this analysis of clinical data.

\section{Patient consent for publication}

Not applicable. 


\section{Competing interests}

The authors declare that they have no competing interests.

\section{References}

1. Rouprêt M, Zigeuner R, Palou J, Boehle A, Kaasinen E, Sylvester R, Babjuk M and Oosterlinck W: European guidelines for the diagnosis and management of upper urinary tract urothelial cell carcinomas: 2011 update. Eur Urol 59: 584-594, 2011.

2. Tawfiek ER and Bagley DH: Upper-tract transitional cell carcinoma. Urology 50: 321-329, 1997.

3. Zini L, Perrotte P, Capitanio U, Jeldres C, Shariat SF, Antebi E, Saad F, Patard JJ, Montorsi F and Karakiewicz PI: Radical versus partial nephrectomy: Effect on overall and noncancer mortality. Cancer 115: 1465-1471, 2009.

4. Go AS, Chertow GM, Fan D, McCullouch CE and Hsu CY: Chronic kidney disease and the risks of death, cardiovascular events and hospitalization. N Engl J Med 351: 1296-1305, 2004.

5. Campbell SC, Novick AC, Belldegrun A, Blute ML, Chow GK, Derweesh IH, Faraday MM, Kaouk JH, Leveillee RJ, Matin SF, et al: Guideline for management of the clinical T1 renal mass. J Urol 182: 1271-1279, 2009.

6. Munoz JJ and Ellison LM: Upper tract urothelial neoplasms: Incidence and survival during the last 2 decades. J Urol 164: 1523-1525, 2000.

7. Siegel R, Naishadham D and Jemal A: Cancer statistics, 2012. CA Cancer J Clin 62: 10-29, 2012.

8. Czito B, Zietman A, Kaufman D, Skowronski U and Shipley W: Adjuvant radiotherapy with and without concurrent chemotherapy for locally advanced transitional cell carcinoma of the renal pelvis and ureter. J Urol 172: 1271-1275, 2004.

9. Epstein JI, Amin MB, Reuter VR and Mostofi FK: The World Health Organization/International Society of Urological Pathology consensus classification of urothelial (transitional cell) neoplasms of the urinary bladder. Bladder consensus conference committee. Am J Surg Pathol 22: 1435-1448, 1998.

10. Kaag MG, O'Malley RL, O'Malley P, Godoy G, Chen M, Smaldone MC, Hrebinko RL, Raman JD, Bochner B, Dalbagni G, et al: Changes in renal function following nephroureterectomy may affect the use of perioperative chemotherapy. Eur Urol 58: 581-587, 2010.

11. Lane BR, Smith AK, Larson BT, Gong MC, Campbell SC, Raghavan D, Dreicer R, Hansel DE and Stephenson AJ: Chronic kidney disease after nephroureterectomy for upper tract urothelial carcinoma and implications for the administration of perioperative chemotherapy. Cancer 116: 2967-2973, 2010 .

12. Lehmann J, Suttmann H, Kovac I, Hack M, Kamradt J, Siemer S, Wullich B, Zwergel U and Stöckle M: Transitional cell carcinoma of the ureter: Prognostic factors influencing progression and survival. Eur Urol 51: 1281-1288, 2007.

13. Arancibia MF, Bolenz C, Michel MS, Keeley FX Jr and Alken P: The modern management of upper tract urothelial cancer: Surgical treatment. BJU Int 99: 978-981, 2007

14. Iborra I, Solsona E, Casanova J, Ricós JV, Rubio J and Climent MA: Conservative elective treatment of upper urinary tract tumors: A multivariate analysis of prognostic factors for recurrence and progression. J Urol 169: 82-85, 2003.
15. Pohar KS and Sheinfeld J: When is partial ureterectomy acceptable for transitional-cell carcinoma of the ureter? J Endourol 15: 405-408, 2001.

16. Yoshida S, Masuda H, Ishii C, Saito K, Kawakami S and Kihara K: Initial experience of functional imaging of upper urinary tract neoplasm by diffusion-weighted magnetic resonance imaging. Int J Urol 15: 140-143, 2008.

17. Sudakoff GS, Dunn DP, Guralnick ML, Hellman RS, Eastwood D and See WA: Multidetector computerized tomography urography as the primary imaging modality for detecting urinary tract neoplasms in patients with asymptomatic hematuria. J Urol 179: 862-867, 2008

18. Lucas SM, Svatek RS, Olgin G, Arriaga Y, Kabbani W, Sagalowsky AI and Lotan Y: Conservative management in selected patients with upper tract urothelial carcinoma compares favourably with early radical surgery. BJU Int 102: 172-176, 2008.

19. Mazeman E: Tumors of the upper excretory urinary tract, calices, renal pelvis and ureter. J Urol 78 (Suppl 9): S1-S219, 1972 (In French)

20. Zincke H and Neves RJ: Feasibility of conservative surgery for transitional cell cancer of the upper urinary tract. Urol Clin North Am 11: 717-724, 1984.

21. Zungri E, Chechile G, Algaba F, Diaz I, Vilá F and Castro C: Treatment of transitional cell carcinoma of the ureter: Is the controversy justified? Eur Urol 17: 276-280, 1990.

22. Lughezzani G, Jeldres C, Isbarn H, Sun M, Shariat SF, Alasker A, Pharand D, Widmer H, Arjane P, Graefen M, et al: Nephroureterectomy and segmental ureterectomy in the treatment of invasive upper tract urothelial carcinoma: A population-based study of 2299 patients. Eur J Cancer 45: 3291-3297, 2009.

23. Bagrodia A, Kuehhas FE, Gayed BA, Wood CG, Raman JD, Kapur P, Derweesh IH, Bensalah K, Sagalowsky AI, Shariat SF, et al: Comparative analysis of oncologic outcomes of partial ureterectomy vs. radical nephroureterectomy in upper tract urothelial carcinoma. Urology 81: 972-977, 2013.

24. Huang Z, Zhang X, Zhang X, Li Q, Liu S, Yu L and Xu T: Segmental ureterectomy is acceptable for high-risk ureteral carcinoma comparing to radical nephroureterectomy. J Invest Surg 25: 1-8, 2018

25. Ho KL and Chow GK: Ureteroscopic resection of upper-tract transitional-cell carcinoma. J Endourol 19: 841-848, 2005.

26. Anderström C, Johansson SL, Pettersson S and Wahlqvist L: Carcinoma of the ureter: A clinicopathological study of 49 cases. J Urol 142: 280-283, 1989.

27. Giannarini G, Schumacher MC, Thalmann GN, Bitton A Fleischmann A and Studer UE: Elective management of transitional cell carcinoma of the distal ureter: Can kidney-sparing surgery be advised? BJU Int 100: 264-268, 2007.

28. Mazeman E: Tumors of the upper urinary tract calyces, renal pelvis and ureter. Eur Urol 2: 120-126, 1976.

29. Leitenberger A, Beyer A and Altwein JE. Organ-sparing treatment for ureteral carcinoma? Eur Urol 29: 272-278, 1996.

30. Uberoi J, Harnisch B, Sethi AS, Babayan RK and Wang DS: Robot-assisted laparoscopic distal ureterectomy and ureteral reimplantation with psoas hitch. J Endourol 21: 368-373, 2007. 